\title{
The diagnostic value of bronchoalveolar lavage and transbronchial lung biopsy in cryptogenic
}

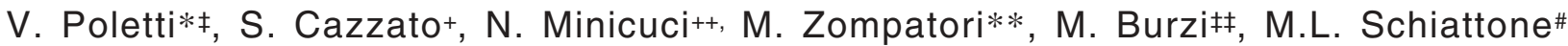

The diagnostic value of bronchoalveolar lavage and transbronchial lung biopsy in cryptogenic organizing pneumonia. V. Poletti, S. Cazzato, N. Minicuci, M. Zompatori, M. Burzi, M.L. Schiattone. (OERS Journals Ltd 1996.

ABSTRACT: In order to determine the diagnostic value of bronchoalveolar lavage (BAL) and transbronchial lung biopsy (TBLB) in cryptogenic organizing pneumonia (COP) a prospective study was carried out.

Thirty seven consecutive patients (20 males and 17 females) with clinicoradiological features of COP were enrolled in the study. The statistical analyses were completed in 35 cases. Twenty eight patients were diagnosed to have COP, all of them with a confirmatory biopsy. In seven cases, a different diagnosis was made. BAL cytological and phenotypical criteria considered for the diagnosis of COP were: a lymphocytosis of more than $25 \%$ (with a CD4/CD8 ratio less than 0.9 ); combined with at least two of the following data (foamy macrophages of $>\mathbf{2 0} \%$, and/or neutrophils of $>5 \%$, and/or eosinophils of $>2 \%$ and $<25 \%$ ). TBLB specimens were classified as positive for COP if they showed: buds of granulation tissue within the centrilobular air spaces; infiltration of alveolar walls with chronic inflammatory cells; and preservation of alveolar architecture.

BAL was performed in 34 patients; 17 cases were consistent with the final diagnosis of COP (sensitivity $63 \%$ ), and four cases were correctly classified as negative (specificity 57\%). BAL had a positive predictive value (PPV) of $85 \%$ and a negative predictive value (NPV) of $29 \%$. TBLB was performed in 32 patients; it correctly identified COP in 16 cases (sensitivity 64\%), and six cases were correctly classified as negative (specificity $86 \%$ ). TBLB had a PPV of $94 \%$ and a NPV of $40 \%$. The accuracy of the examinations, that is the probability of correctly diagnosing both diseased and nondiseased patients by BAL or TBLB, was 62 and $69 \%$, respectively.

Our findings suggest that the combination of cytological bronchoalveolar lavage and histological transbronchial lung biopsy data obtained during a fibreoptic procedure appears to be an effective method for the initial investigation in cryptogenic organizing patients pneumonia presenting with patchy radiographic shadows. Eur Respir J., 1996, 9, 2513-2516.
*Divisione di Pneumologia and **Servizio di Radiologia Pneumo-Nefro, Policlinico S. Orsola-Malpighi, Bologna, Italy. ‡Divisione di Pneumologia, $\$$ Servizio di Radiologia, and "Patologia Clinica, Ospedale Maggiore, Bologna, Italy. ${ }^{+}$Clinica Pediatrica, Università di Bologna, Italy. ${ }^{++}$Statistic Consultant, Università di Padova, Italy.

Correspondence: V. Poletti Divisione di Pneumologia Laboratorio di Patologia Polmonare PoliClinico S. Orsola-Malpighi Via P. Palagi 9 40138, Bologna Italy

Keywords: Bronchoalveolar lavage bronchiolitis obliterans

cryptogenic organizing pneumonia diagnosis

transbronchial lung biopsy

Received: October 261995

Accepted after revision August 161996
Cryptogenic organizing pneumonia (COP) or bronchiolitis obliterans-organizing pneumonia (BOOP) is a distinct clinicopathological entity of unknown cause, which usually responds significantly to corticosteroids [1-6]. The clinical features of COP are characterized by persistent, nonproductive cough and frequently, a flulike illness with fever, malaise, a short history of progressive dyspnoea, associated, in most cases, with patchy peripheral infiltrates on a chest radiogram or a computerized tomographic (CT) scan [7, 8]. Histologically, it is defined by the presence of buds of granulation tissue (Masson bodies) in alveoli and alveolar ducts, the infiltration of alveolar walls with chronic inflammatory cells and the preservation of alveolar architecture. Histopathological features of COP are nonspecific and may be seen in a number of pulmonary repair reactions [9]. Therefore, it is essential to maintain stringent clinical criteria in order to exclude all other diseases that can imitate COP clinically and morphologically. We undertook the present study to evaluate the diagnostic results of bronchoalveolar lavage (BAL) and transbronchial lung biopsy (TBLB) in COP. In this context, we carried out a prospective study of the usefulness of BAL and TBLB in patients with suspected COP.

\section{Materials and methods}

\section{Patient population}

Criteria for admission to the study were: 1) a subacute history of cough and/or progressive dyspnoea with or without malaise, weight loss, fever or a pattern of flu-like illness; 2) crackles upon physical examination; 3) abnormal gas exchange; 4) bilateral patchy infiltrates on chest radiographs; 5) negative microbiological analyses on BAL fluid; and 6) serological assays negative for Mycoplasma pneumoniae, Chlamydia trachomatis and 
Chlamydia pneumoniae, Legionella pneumophila, Coxiella burnetii, adenovirus, respiratory syncyitial virus, cytomegalovirus, herpes simplex, influenzae and parainfluenzae viruses.

A prospective analysis was made of 37 consecutive patients (20 males and 17 females), admitted to our department with clinical and radiological features suggestive of COP according to the above-mentioned criteria. The average age was 60 yrs (range 20-79 yrs). None of the patients were in need of mechanical ventilation. At the time of diagnosis, none of the patients in the study were being treated with corticosteroids. All patients underwent fibreoptic bronchoscopy.

\section{Bronchoscopic technique}

After informed consent on the part of the patients, a flexible fibreoptic bronchoscopy was performed with an Olympus BF-B3 bronchoscope, following the application of topical lidocaine. BAL was performed in 36 patients (one patient with an ischaemic cardiomyopathy did not undergo BAL in order to avoid pulmonary oedema) by the instillation of $150 \mathrm{~mL}$ of $0.9 \%$ saline in $6 \times 25$ aliquots, either into the segment involved when the lesion was localized or into middle lobe when shadowing was diffuse.

\section{Bronchoalveolar lavage and transbronchial lung biop- sy procedures}

The fluid recovered was filtered through surgical gauze and the volume was measured. The first aliquot of BAL fluid was used for microbiological studies. All subsequent aliquots were pooled together for processing. The total counts were determined by haemocytomer. Cell differentials were numbered from cytospin preparations stained with Diff-Quick by counting at least 400 cells. Eight cytospin slides fixed in alcohol and stained with the Papanicolaou method were screened for neoplastic cells. The lymphocyte subpopulations were determined by using a flow cytometer after staining with monoclonal antibodies as described previously [10]. The BAL findings were arbitrarily classified as positive for COP if they showed a lymphocytosis of more than $25 \%$ (with a CD4/CD8 ratio $<0.9$ ) combined with at least two of the following criteria: foamy macrophages $>20 \%$, and/or neutrophils of $>5 \%$ and/or eosinophils $>2 \%$ and $<25 \%$. BAL data were considered evaluable only when the fluid recovered was more than $40 \%$.

All patients underwent TBLB, with the exception of three patients who agreed to undergo only BAL. TBLB and $\mathrm{BAL}$ were carried out during the same bronchoscopic examination. Biopsy specimens were processed as described previously [11]. At least five, and usually six, specimens were obtained. They were stored in a $10 \%$ buffered formalin solution and embedded in paraffin. Five slides were prepared from serial sections of each block and were stained with haematoxylin and eosin, Ziehl-Neelsen, periodic-acid-Schiff, Giemsa, Gomori's methenamine silver, and van Gieson's elastic fibres. The TBLB specimens were classified as being positive for COP if they showed buds of granulation tissue (Masson bodies) within the small airways and alveoli, the infiltration of alveolar walls with chronic inflammatory cells, and the preservation of alveolar architecture [12].
Table 1. - Diagnosis of the 35 patients

\begin{tabular}{lr}
\hline Disease & $\mathrm{n}$ \\
\hline COP & 28 \\
Chronic eosinophilic pneumonia & 2 \\
Metastatic kidney adenocarcinoma & 1 \\
Primary large cell anaplastic lymphoma & 1 \\
Tuberculosis & 1 \\
Bronchoalveolar carcinoma & 2 \\
\hline Total & 35 \\
\hline
\end{tabular}

COP: cryptogenic organizing pneumonia.

All BAL data and biopsy slides were evaluated independently, without any knowledge of clinical and laboratory data.

\section{Diagnostic criteria}

Of the 37 patients, two were excluded as the BAL recovery was less than $40 \%$ and they refused to be submitted to a lung biopsy. In one patient BAL was not performed in order to avoid cardiopulmonary complications, and he was submitted only to TBLB.

In the present study 28 patients were diagnosed as having COP on the basis of: 1) bioptic proof of intraalveolar organizing or fibrotic buds (documented by TBLB in 16 cases, by open lung biopsy in 11 cases and by transthoracic lung biopsy in one case); and 2) a favourable clinical course after corticosteroid treatment.

In seven cases, a different diagnosis was made: two bronchoalveolar carcinomas (morphologically documented by TBLB in both cases and by BAL in one case); two cases of chronic eosinophilic pneumonia (documented by TBLB and BAL); one case of metastatic adenocarcinoma of the kidney (documented by transthoracic biopsy); one case of large cell anaplastic lymphoma in the lung (documented by open lung biopsy) and, finally, one case of tuberculosis diagnosed by transthoracic biopsy (table 1).

\section{Statistical analysis}

The sensitivity, specificity, positive predictive value (PPV), negative predictive value (NPV) and accuracy of each of the two diagnostic tests (BAL and TBLB) were calculated. A statistical analysis of the results, when appropriate, was performed using the Chi-squared test with Yates correction for continuity and Fisher's exact test. A p value less than 0.05 was considered significant.

\section{Results}

BAL fluid analysis was carried out in 34 patients (table $2)$. Seventeen cases were considered consistent with the final diagnosis of COP (sensitivity 63\%). Four cases were correctly classified as negative (specificity 57\%). The PPV of BAL was $85 \%$, and the NPV $29 \%$ (table 3 ).

TBLB was performed in 32 patients (table 2). It correctly identified COP in 16 cases (sensitivity 64\%), and six cases were correctly classified as negative (specificity $86 \%$ ). TBLB had a PPV of $94 \%$, and a NPV of $40 \%$. 
Table 2. - Results of the diagnostic procedures used in 35 patients with clinical and radiologic features suggestive of COP

\begin{tabular}{|c|c|c|c|}
\hline $\begin{array}{l}\mathrm{Pt} \\
\text { No. }\end{array}$ & $\begin{array}{c}\text { BAL } \\
\text { cytology }\end{array}$ & TBLB & $\begin{array}{c}\text { Final } \\
\text { diagnosis }\end{array}$ \\
\hline 1 & - & + & COP \\
\hline 2 & - & - & $\mathrm{COP}$ \\
\hline 3 & + & - & COP \\
\hline 4 & + & + & $\mathrm{COP}$ \\
\hline 5 & + & - & $\mathrm{COP}$ \\
\hline 6 & + & NP & COP \\
\hline 7 & + & - & $\mathrm{COP}$ \\
\hline 8 & + & + & $\mathrm{COP}$ \\
\hline 9 & + & NP & $\mathrm{COP}$ \\
\hline 10 & - & + & $\mathrm{COP}$ \\
\hline 11 & - & NP & COP \\
\hline 12 & + & + & COP \\
\hline 13 & + & - & $\mathrm{COP}$ \\
\hline 14 & + & + & $\mathrm{COP}$ \\
\hline 15 & + & + & $\mathrm{COP}$ \\
\hline 16 & - & - & COP \\
\hline 17 & + & - & COP \\
\hline 18 & + & - & COP \\
\hline 19 & - & + & $\mathrm{COP}$ \\
\hline 20 & - & + & $\mathrm{COP}$ \\
\hline 21 & + & + & $\mathrm{COP}$ \\
\hline 22 & + & + & $\mathrm{COP}$ \\
\hline 23 & + & + & COP \\
\hline 24 & - & + & $\mathrm{COP}$ \\
\hline 25 & + & + & COP \\
\hline 26 & - & - & $\mathrm{COP}$ \\
\hline 27 & - & + & $\mathrm{COP}$ \\
\hline 28 & NP & + & COP \\
\hline 29 & + & - & Bronchoalveolar carcinoma \\
\hline 30 & - & - & Bronchoalveolar carcinoma \\
\hline 31 & + & + & Metastatic kidney adenocarcinoma \\
\hline 32 & + & - & Primary large cell lymphoma \\
\hline 33 & - & - & Chronic eosinophilic pneumonia \\
\hline 34 & - & - & Chronic eosinophilic pneumonia \\
\hline 35 & - & - & Tuberculosis \\
\hline
\end{tabular}

COP: cryptogenic organizing pneumonia; BAL: bronchoalveolar lavage; TBLB: transbronchial lung biopsy; NP: not performed; +: positive for COP; -: negative for COP.

The accuracy, that is the probability of correctly diagnosing both diseased and nondiseased patients, of BAL and TBLB was 62 and 69\%, respectively (table 3). In the group of patients affected by COP, both procedures combined were used in 24 patients, and were diagnostic in 21 patients (sensitivity $88 \%$ ). There were three false positive cases after BAL examination. One patient had metastatic kidney adenocarcinoma. The other two patients had, respectively, bronchioloalveolar carcinoma and anaplastic large cell lymphoma primary of the lung. TBLB gave only one false-positive result in a patient with adenocarcinoma of the kidney (table 2).

The diagnostic yield of TBLB proved to be similar to that of BAL (sensitivity 64 versus $63 \%$ ). The combination of the two procedures (BAL and TBLB) produced a greater percentage of correct positive diagnoses (sensitivity $88 \%)$ than BAL or TBLB alone $(\mathrm{p}<0.05)$. In fact, TBLB made it possible to confirm the diagnosis in seven cases in which BAL was considered nondiagnostic (six cases) or was not performed (one case). BAL profile was consistent with the clinical diagnosis of COP in six patients in which TBLB specimens were not trustworthy and in two cases in which TBLB was not performed.

\section{Discussion}

The diagnosis of COP is based on the combination of characteristic clinical and roentgenological features, with nonspecific histological findings (intra-alveolar organizing or fibrotic buds, chronic interstitial alveolitis, and preservation of the alveolar architecture). An open lung biopsy is considered to be the best way to obtain a representative lung specimen. However, transbronchial biopsy has been reported to be sufficient as far as the confirmation is concerned $[8,12,13]$. Recently, BAL has been thought to have a characteristic cytological and phenotypical profile in this disease $[6,14]$.

The purpose of this prospective study was to assess the value of BAL and TBLB in the diagnosis of COP. A well-defined group of patients with a presumed diagnosis of COP based on clinical and roentgenological features took part in the study. They were all examined consecutively in our department. None of them were being treated with corticosteroids at the time of the study, and none were in need of mechanical ventilation. All of the patients showed patchy alveolar infiltrates on chest radiographic image and none of them presented any signs of underlying disorder or known causes associated with COP.

In this study, 28 of the 35 patients evaluated were affected by COP $(80 \%)$. All of them showed a dramatic response to corticosteroid therapy. In this group of patients, BAL was performed in 27 cases and was positive in agreement with our arbitrary criteria in $17(63 \%)$. Thus, the sensitivity of BAL was $63 \%$, and the specificity was $57 \%$. In fact, there were three false-positive diagnoses of COP from cytological studies of BAL fluid. In one patient, the histological study on TBLB specimens demonstrated a bronchioloalveolar carcinoma. The second patient had a diagnosis of lymphoma in the samples obtained during a video-assisted thoracoscopy. In the third patient, both procedures, BAL and TBLB, gave false-positive results for COP. In this patient, on total body CT scan a kidney lesion was seen, suggesting a different diagnosis. Therefore, a transthoracic lung biopsy was performed and it identified a metastatic adenocarcinoma of the kidney.

In this study, the sensitivity of TBLB $(64 \%)$ was found to be similar to BAL, and the specificity better ( $86 \mathrm{vs}$ $57 \%$ ), although not at a statistically significant level.

Table 3. - Statistical analysis of the performance of two diagnostic tests in the evaluation of COP

\begin{tabular}{lcccccccccc}
\hline $\begin{array}{l}\text { Diagnostic } \\
\text { test }\end{array}$ & $\begin{array}{c}\text { Pts } \\
\mathrm{n}\end{array}$ & $\begin{array}{c}\text { True } \\
+\mathrm{ve}\end{array}$ & $\begin{array}{c}\text { True } \\
-\mathrm{ve}\end{array}$ & $\begin{array}{c}\text { False } \\
+\mathrm{ve}\end{array}$ & $\begin{array}{c}\text { False } \\
-\mathrm{ve}\end{array}$ & Specificity & Sensitivity & PPV & NPV & Accuracy \\
\hline BAL & 34 & 17 & 4 & 3 & 10 & 0.57 & 0.63 & 0.85 & 0.29 & 0.62 \\
TBLB & 32 & 16 & 6 & 1 & 9 & 0.86 & 0.64 & 0.94 & 0.40 & 0.69
\end{tabular}

PPV: positive predictive value; NPV: negative predictive value; +ve: positive; -ve: negative; BAL: bronchoalveolar lavage; TBLB: transbronchial lung biopsy; COP: cryptogenic organizing pneumonia. 
The BAL pattern in COP patients has been reported to be characteristic. Most authors agree that there is a striking increase of lymphocytes [6, 14, 15]. A neutrophilic increase in BAL fluid has been reported only in a subset of patients with a rapidly progressive form of COP [16]. Costabel and co-workers [14], first mentioned that the BAL profile in COP patients is characterized by several features: conspicuous cell differentials with an increase in all cell types, most markedly in lymphocytes and more moderately in neutrophils, eosinophils and mast cells; the presence of foamy macrophages and, occasionally, of plasma cells; and a decreased CD4 CD8 ratio. They suggested this pattern might be of value to distinguish between COP and other interstitial lung disease (extrinsic allergic alveolitis, chronic eosinophilic pneumonia, interstitial pulmonary fibrosis). The value of a method of pattern analysis in order to render a clinically meaningful diagnosis is well-known by pathologists and mostly by dermatopathologists [17]. In the present study, we chose arbitrary cut-off levels for the BAL percentages of cells (coupled with a CD4/CD8 ratio $<0.9$ ) to consider the test as positive for COP, based on the references mentioned $[6,14,15]$ and our experience.

TBLB has been sown to be an extremely safe and effective method for obtaining lung tissue. The presence of Masson bodies within alveoli and bronchioles, with preservation of the underlying architecture, is the essential histological feature which is necessary for a diagnosis of COP (if the clinical findings are appropriate) [12]. In this series, we had one patient with proven metastatic tumour in the lung that presented intra-alveolar organizing or fibrotic buds as the only morphological lesion on TBLB samples. Intra-alveolar fibrosis is a well-known nonspecific morphological pattern. It is characteristic of COP but may be seen in a number of lung disorders: organizing infections; organizing diffuse alveolar damage; allergic and/or drug reactions; collagen-vascular disease; organizing toxic exposure; eosinophilic pneumonia; histiocytosis X; Wegener's syndrome; and around other lesions, including abscesses and tumours $[9,18]$. Transbronchial lung biopsy specimens are tiny and unselected [11]. However, a morphological diagnosis of organizing pneumonia can be established by TBLB. The adequacy of the biopsy (number of specimens and number of centrilobular airway structures) is important in order to avoid misdiagnosis [12]. Dina and SHEPPARD [12] reported that a histological diagnosis of COP on TBLB specimens was sufficient to confirm the clinical hypothesis in 7 out of 11 cases $(64 \%)$, which is in agreement with the present study.

In conclusion, our findings suggest that the combination of cytological (bronchoalveolar lavage) and histological (transbronchial lung biopsy) procedures appear to be an effective method for initial investigation in cryptogenic organizing pneumonia patients presenting with patchy radiographic shadows. This diagnostic approach has also been accepted in other morphologically nonspecific interstitial lung diseases (sarcoidosis, hypersensitivity pneumonitis) $[11,19]$. Our prospective study did not include a high resolution computed tomographic scan. It may be that bronchoscopic procedures targeted by computed tomographic scan could improve the diagnostic value of bronchoalveolar lavage and transbronchial lung biopsy [7, 8].

\section{References}

1. Gosink BB, Friedman PJ, Liebow AA. Bronchiolitis obliterans: roentgenologic-pathologic correlation. Am J Roentgenol 1973; 117: 816-832.

2. Grimblat J, Mechlis S, Lewitus Z. Organizing pneumonia-like process: an unusual observation in steroid responsive cases with features of chronic interstitial pneumonia. Chest 1981; 80: 259-263.

3. Davison AG, Heard BE, Maallister WAC, Turner-Warwich ME. Cryptogenic organizing pneumonia. Q J Med 1983; 52: 382-394.

4. Epler GR, Colby TV, McLoud TC, Carrington CB, Gaensler EA. Bronchiolitis obliterans organizing pneumonia. N Engl J Med 1985; 312: 152-158.

5. Costabel U, Teschler H, Scoenfeld B, et al. BOOP in Europe. Chest 1992; 102 (s): 14-20.

6. King TE, Mortensen RL. Cryptogenic organizing pneumonia. Chest 1992; 102 (s): 8-13.

7. Bouchardy LM, Kuhlman JE, Ball WC, Hruban RH, Askin FB, Siegelman SS. CT findings in bronchiolitis obliterans organizing pneumonia (BOOP) with radiographic, clinical, and histologic correlation. Comput Assist Tomogr 1993; 17: 352-357.

8. Lee KS, Kulling P, Hartman T, Muller NL. Cryptogenic organizing pneumonia: CT findings in 43 patients. AJR 1994; 162: 543-546.

9. Colby TV. Pathologic aspects of bronchiolitis obliterans organizing pneumonia. Chest 1992; 102 (s): 38-43.

10. Agostini C, Poletti V, Zambello R, et al. Phenotypical analysis of bronchoalveolar lavage in patients with HIV infection. Am Rev Respir Dis 1988; 138: 1609-1615.

11. Poletti V, Patelli M, Poggi S, Bertanti T, Spiga L, Ferracini R. Transbronchial lung biopsy and bronchoalveolar lavage in diffuse infiltrative lung disease. Respiration 1988; 54 (s): 66-70.

12. Dina R, Sheppard MN. The histological diagnosis of clinically documented cases of cryptogenic organizing pneumonia: diagnostic features in transbronchial biopsies. Histopathology 1993; 23: 541-545.

13. Bartter T, Irwin RS, Nash G, Balikian JP, Hollingswoorth HH. Idiopathic bronchiolitis obliterans organizing pneumonia with peripheral infiltrates on chest roentgenogram. Arch Intern Med 1989; 149: 273-279.

14. Costabel U, Teschler H, Guzman I. Bronchiolitis obliterans organizing pneumonia (BOOP): the cytological and immunocytological profile of bronchoalveolar lavage. Eur Respir J 1992; 5: 791-797.

15. Cordier JF, Loire R, Brune J. Idiopathic bronchiolitis obliterans organizing pneumonia: definition of characteristic clinical profiles in a series of 16 patients. Chest 1989; 96: 999-1004.

16. Cohen AJ, King TE, Downey GP. Rapidly progressive bronchiolitis obliterans with organizing pneumonia. Am J Respir Crit Care Med 1994; 149: 1670-1675.

17. Ackerman AB. Histologic diagnosis of inflammatory skin disease: a method by pattern analysis. Philadelphia, Lea and Febiger, 1978.

18. Ologade CO, Hotty TB, Douglas WW, Colby TV, Sur S. Chronic eosinophilic pneumonia and idiopathic bronchiolitis obliterans organizing pneumonia: comparison of eosinophil number and degranulation by immunofluorescence staining for eosinophil derived major basic protein. Mayo Clin Proc 1995; 70: 137-142.

19. Rosen Y. Sarcoidosis. In: Dail DH, Hammar SP, eds. Pulmonary Pathology. New York, Springer-Verlag, 1994: pp. 629-633. 